\title{
Nuclear Effects in Hadron Production at HERMES
}

\author{
Nicola Bianchi \\ On behalf of the HERMES Collaboration
}

INFN- Laboratori Nazionali di Frascati.

Via E. Fermi 40, I 00044 Frascati (Italy)

\begin{abstract}
The influence of the nuclear medium on the lepto-production of hadrons was studied in semi-inclusive deep-inelastic scattering off several nuclear targets. In particular, at the HERMES experiment at DESY, the differential multiplicity for nuclei relative to that of deuterium has been measured for the first time for various identified hadrons $\left(\pi^{+}, \pi^{-}, \pi^{0}, K^{+}, K^{-}, p\right.$ and anti-p) as a function of the virtual photon energy $v$, the fraction $z$ of this energy transferred to the hadron, and the hadron transverse momentum squared $p_{t}^{2}$. The distribution of the hadron transverse momentum is broadened towards high $\mathrm{p}_{\mathrm{t}}{ }^{2}$ in the nuclear medium, in a manner resembling the Cronin effect previously observed in collisions of heavy ions and protons with nuclei.
\end{abstract}

Keywords: Nulear Medium, Hadronization, leptoproduction.

PACS: $13.60 . \mathrm{Hb}, 25.30 . \mathrm{Rw}$

In Deep Inelastic Scattering, the fragmentation process can be described by a fragmentation functions $\mathrm{D}_{\mathrm{f}}^{\mathrm{h}}(\mathrm{z})$ in the vacuum, denoting the probability that a quark of flavor $f$ produces a hadron of type $h$ carrying a fraction $z$ of the energy of the struck quark in the target rest frame ${ }^{1)}$. In the nuclear medium additional hard or soft processes may occur before the final-state hadron is completely formed influencing the hadronization process, e.g. causing a change in the quark fragmentation functions. Quark propagation in the nuclear environment involves processes like partonic energy loss, rescattering during the pre-hadronic formation process or interaction of the finalstate hadron within the nucleus. If the final hadron is formed inside the nucleus, the hadron can interact via the relevant hadronic interaction cross section, causing a further reduction of the hadron yield. Therefore, quark and hadron propagation in nuclei are expected to result in a modification i.e. a "softening" of the leading-hadron spectra compared to that from a free nucleon. By studying the properties of the leading-hadron emerging from nuclei, information on the characteristic time-distance scales of hadronization can be also derived.

In-medium modification of the quark fragmentation function has been described in terms of rescattering of gluons and quarks, and of energy loss due to induced gluon radiation $^{2,3)}$. Alternatively, colorless pre-hadron rescattering ${ }^{4,5)}$ in the medium has been

CP964,QCD@WORK 2007, International Workshop on Quantum Chromodynamics: Theory and Experiment edited by P. Colangelo, D. Creanza, F. De Fazio, R. A. Fini, E. Nappi, and G. Nardulli (C) 2007 American Institute of Physics 978-0-7354-0480-9/07/\$23.00 
suggested with additional effects due to $\mathrm{Q}^{2}$ rescaling ${ }^{6)}$. Older interpretations ${ }^{7)}$ based on hadronic final-state interactions require a hadron formation length smaller than the nuclear size, which is unlikely for struck quarks boosted to energies larger than a few $\mathrm{GeV}$. Semi-inclusive deep-inelastic lepton-nucleus collisions are most suitable to obtain quantitative information on the hadronization process. In contrast to hadronnucleus and nucleus-nucleus scattering, in deep-inelastic scattering no deconvolution of the parton distributions of the projectile and target particles is needed, so that hadron distributions and multiplicities from various nuclei can be directly related to nuclear effects in quark propagation and hadronization.

The experimental results for semi-inclusive deep-inelastic scattering on nuclei ${ }^{8-11)}$ are presented in terms of the hadron multiplicity ratio $R_{M}{ }^{h}$, which represents the ratio of the number of hadrons of type $h$ produced per deep-inelastic scattering event on a nuclear target of mass $A$ to that from a deuterium target (D). The ratio $R_{M}{ }^{h}$ depends on the leptonic variables $v$ and $\mathrm{Q}^{2}$, which are the energy in the target rest frame and the squared four-momentum of the virtual photon respectively, and on the hadronic variables $\mathrm{z}=\mathrm{E}_{\mathrm{h}} / \mathrm{v}$ and $\mathrm{p}_{\mathrm{t}}{ }^{2}$, where $\mathrm{p}_{\mathrm{t}}$ is the hadron momentum component transverse to the virtual photon direction. The measurements described were performed with the HERMES spectrometer ${ }^{12)}$ using the $27.6 \mathrm{GeV}$ positron beam stored in the HERA ring at DESY. The hadron identification was accomplished using the information from the RICH detector.

The electromagnetic calorimeter provided neutral pion identification by the detection of neutral clusters originating from the two decay photons. Scattered positrons were selected by imposing the constraints $\mathrm{Q}^{2}>1 \mathrm{GeV}^{2}, \mathrm{~W}>2 \mathrm{GeV}$ for the invariant mass of the photon-nucleon system, and $y=v / E<0.85$ for the energy fraction of the virtual photon. Contributions from target fragmentation were suppressed by requiring $z>0.2$.

The data have been corrected for radiative processes involving nuclear elastic, quasi-elastic and inelastic scattering. The size of the radiative corrections applied to $\mathrm{R}_{\mathrm{M}}^{\mathrm{h}}$ was found to be negligible in most of the kinematical range, with a maximum of about $7 \%$ at the highest value of $v$, as most of the radiative contributions cancel in the multiplicity ratio. The systematic uncertainty is reduced due to the fact that multiplicity ratios of semi-inclusive and inclusive yields are measured.

The multiplicity ratio has been determined as a function of $z, v, \mathrm{Q}^{2}$ or $\mathrm{p}_{\mathrm{t}}{ }^{2}$, while integrating over all other kinematical variables. Figures 1 and 2 show some of the HERMES data for $\mathrm{R}_{\mathrm{M}}{ }^{\mathrm{h}}$ for different final state hadrons, for different nuclei and for different kinematical variables. As it is seen larger medium effects are found for heavier nuclei, while remarkable differences are present between different types of hadrons, in particular for the proton case. The effects are larger for low $\mathrm{v}$, high $\mathrm{z}$ and low $\mathrm{Q}^{2}$. Results for neutral pions are in agreement with the ones for charged pions. 


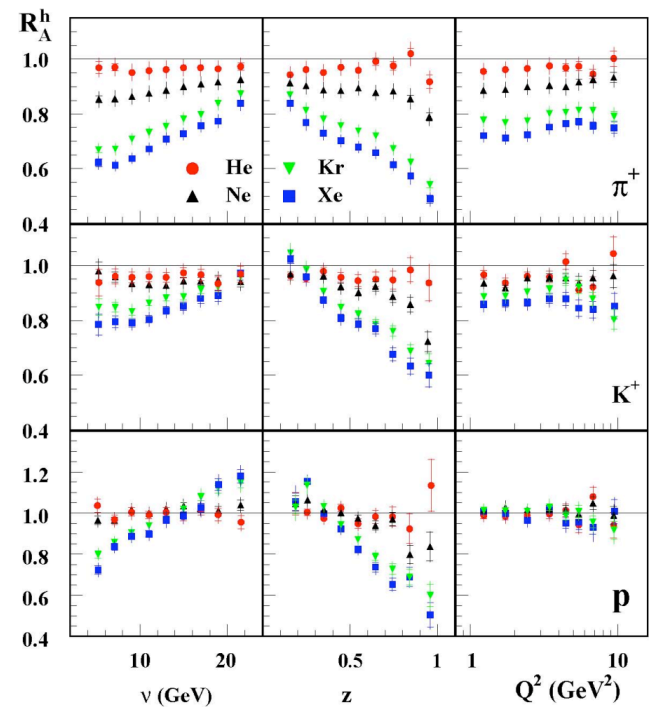

FIGURE 1. HERMES data for positive charged hadrons, as a function of $v, z$ and $\mathrm{Q}^{2}$.

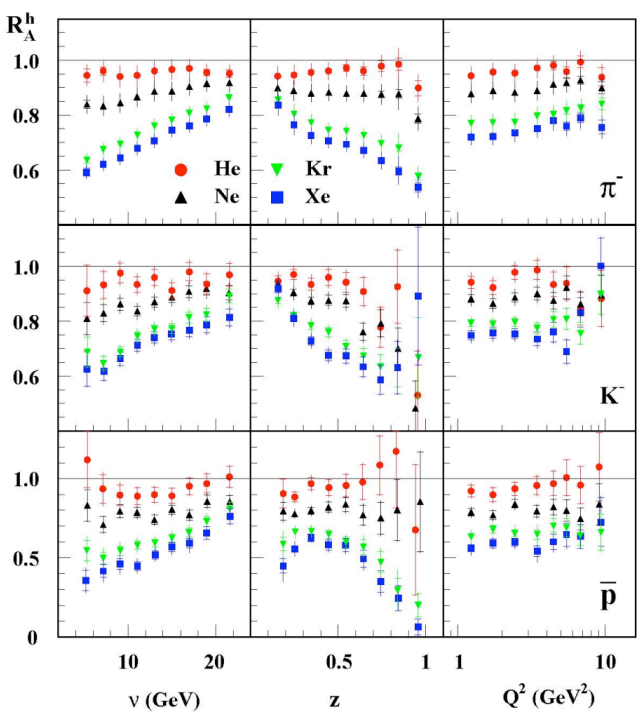

FIGURE 2. HERMES data for negative charged hadrons, as a function of $v, \mathrm{z}$ and $\mathrm{Q}^{2}$

The Cronin effect, like in the case of heavy ion collision, is also observed as an enhancement of hadrons at large $\mathrm{p}_{\mathrm{t}}$ (see Figure 3). Again the effect is different for different hadrons. 


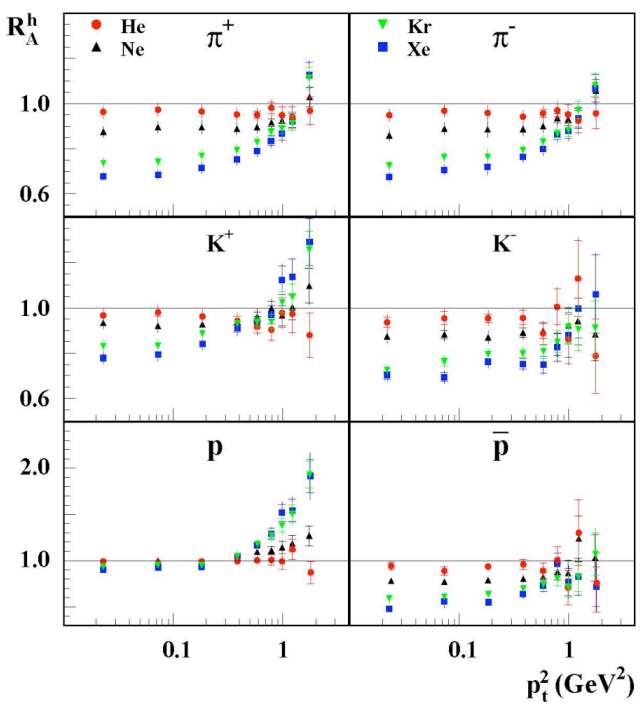

FIGURE 3. HERMES data for charged hadrons as a function of $\mathrm{p}_{\mathrm{t}}{ }_{\mathrm{t}}$.

The $\mathrm{p}_{\mathrm{t}}^{2}$ distribution of the observed hadrons, with respect to the virtual photon direction, is expected to be broadened on a nuclear target compared to a proton or a deuteron target due to multiple scattering of the propagating quark and hadron. To better study this process, the absolute difference between the transverse momentum of the emerging hadron produced in a nucleus with respect to the one produced in the deuterium has been also measured. In Figure 4 preliminary data are presented for different hadrons.

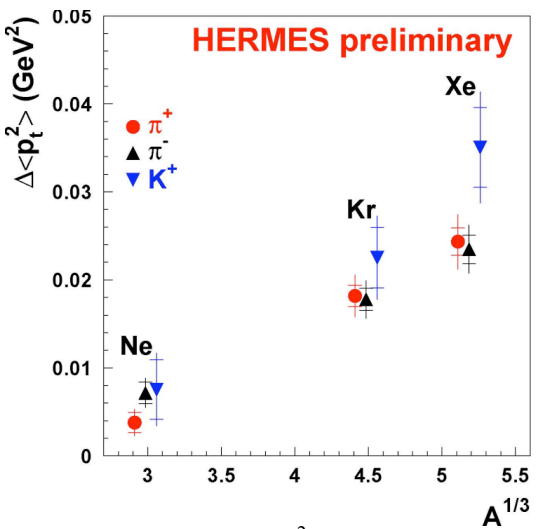

FIGURE 4. HERMES preliminary data for the $\mathrm{p}_{\mathrm{t}}{ }^{2}$ broadening as function of the mass number. 
It is to point out that, while the measured hadron attenuation at low $v$ and high $\mathrm{z}$ is compatible with an increase which follows an $\mathrm{A}^{2 / 3}$ law, the measured $\mathrm{p}_{\mathrm{t}}$ broadening is compatible with an $A^{1 / 3}$ law. Since the nuclear size scales approximately with $A^{1 / 3}$, the hadron attenuation may be interpreted by a coherent process like the partonic energy loss via in medium forward gluon emission (LPM QCD effect), while the hadron $p_{t}$ broadening by an incoherent process like the in medium partonic rescattering.

From what above, the essential features of the HERMES data support a partonic interpretation of the medium effect in the hadronization process, but a contribution of pre-hadronic interactions may still be present in the relevant kinematic range. Therefore the HERMES results on medium modification of fragmentation functions are also essential for a correct understanding of the nuclear effects in heavy ion collision obtained at SPS, RHIC and which will be soon available at LHC, since for cold static nuclei, as the ones probed by DIS, the medium density and size are known and the initial state interactions are absent. In particular the increase of the medium density in the hot fireball generated at the early stage of the high energy heavy ion collision can be inferred from the relative hadronic and jet quenching with respect to DIS data.

\section{REFERENCES}

1. HERMES Coll., A. Airapetian et al., Eur. Phys.J. C 2 , 599 (2001).

2. F. Arleo, Nucl. Phys. A 715, 899 (2003) .

3. X.N. Wang and X. Guo, Nucl. Phys. A 696,788 (2001); E. Wang and X.N. Wang, Phys. Rev. Lett. 89, 162301 (2002).

4. T. Falter et al., Phys. Lett. B 594, 61 (2004); Phys. Rev. C 70, 054609 (2004); K. Gallmeister and W. Cassing, Nucl. Phys. A 748, 241 (2005); K. Gallmeister and T. Falter, Phys. Lett. B 630, 40 (2005).

5. B.Z.Kopeliovich, J.Nemchik, E.Predazzi, A.Hayashigaki, Nucl. Phys. A 740, 211 (2004).

6. A. Accardi, V. Muccifora, H.J. Pirner, Nucl. Phys. A 720, 131 (2003) .

7. A. Bialas, Acta Phys. Pol. B 11, 475 (1980).

8. HERMES Coll., A. Airapetian et al., Eur. Phys. J. C 20, 479(2001).

9. HERMES Coll., A. Airapetian et al., Phys. Lett. B 57, 37 (2003).

10. HERMES Coll., A. Airapetian et al., Phys. Rev. Lett. 96, 162301 (2006).

11. HERMES Coll., A. Airapetian et al., Nucl. Phys. B 780, 1 (2007).

12. HERMES Coll., K. Ackerstaff et al., Nucl. Instr. and Meth. A 41, 230 (1998). 